\title{
SECONDARY BROWN-KERVAIRE QUADRATIC FORMS AND $\pi$-MANIFOLDS
}

\author{
FUQUAN FANG AND JIANZHONG PAN
}

\begin{abstract}
In this paper we assert that for each $\Phi$-oriented $2 n$ manifold (c.f : Definition 1.1) $M$ where $n \geq 4$ and $n \neq 3(\bmod 4)$, there is a well-defined quadratic function $\phi_{M}: H^{n-1}\left(M, \mathbb{Z}_{4}\right) \rightarrow$ $\mathbb{Q} / \mathbb{Z}$, we call the secondary Brown-Kervaire quadratic forms, so that

- $\phi_{M}(x+y)=\phi_{M}(x)+\phi_{M}(y)+j\left(x \cup S q^{2} y\right)[M]$,

- the Witt class of $\phi_{M}$ is a homotopy invariant, if the Wu class $v_{n+2-2^{i}}\left(\nu_{M}\right)=0$ for all $i$.

where $j: \mathbb{Z}_{2} \rightarrow \mathbb{Q} / \mathbb{Z}$ is the inclusion homomorphism and $\nu_{M}$ the stable normal bundle of $M$.

Among the applications we obtain a complete classification of $(n-2)$-connected $2 n$-dimensional $\pi$-manifolds up to homeomorphism and homotopy equivalence, where $n \geq 4$ and $n+2 \neq 2^{i}$ for any $i$. In particular, we prove that the homotopy type of such manifolds determine their homeomorphism type.
\end{abstract}

\section{INTRODUCTION}

Let $M$ be a $2 n$-dimenisonal framed manifold (i.e. a $\pi$-manifold with a framing) where $n=1(\bmod 2)$. The Kervaire invariant of $M$ is the Arf invariant of a $\mathbb{Z}_{2}$-valued Kervaire quadratic form of $M$

$$
q_{M}: H^{n}\left(M, \mathbb{Z}_{2}\right) \rightarrow \mathbb{Z}_{2}
$$

satisfying

$$
q_{M}(x+y)=q_{M}(x)+q_{M}(y)+(x \cup y)[M]_{2}
$$

It was invented by Kervaire to find the first example of non-smoothable PL-manifold. Kervaire invariants and its various generalizations, e.g.

Date: Dec. 20,1999.

The first author was supported in part by NSFC 1974002, Qiu-Shi Foundation and $\mathrm{CNPq}$ and the second author is partially supported by the NSFC project 19701032 and ZD9603 of Chinese Academy of Science and Brain Pool program of KOSEF . 
the Brown-Kervaire invariants[4], play very important roles in geometric topology. Formally, $q_{M}$ is a "quadratic form" subject to the symmetric bilinear form

$$
\begin{gathered}
H^{n}\left(M, \mathbb{Z}_{2}\right) \times H^{n}\left(M, \mathbb{Z}_{2}\right) \rightarrow \mathbb{Z}_{2} \\
(x, y) \rightarrow x \cup y[M]_{2}
\end{gathered}
$$

For a Spin manifold of even dimension, there is another symmetric bilinear form $\mu_{M}$ studied by Landweber and Stong [16]:

$$
\begin{gathered}
\mu_{M}: H^{n-1}\left(M, \mathbb{Z}_{2}\right) \times H^{n-1}\left(M, \mathbb{Z}_{2}\right) \rightarrow \mathbb{Z}_{2} \\
(x, y) \rightarrow S q^{2}(x) \cup y[M]_{2}
\end{gathered}
$$

A natural algebraic question to ask is whether there is an intrinsic "quadratic form" of $M$ subject to $\mu_{M}$. To answer this turns out to be the main novelty of this paper. For a large family of Spin manifolds including all $\pi$-manifolds, the so called $\Phi$-oriented manifolds, we will define a $\mathbb{Q} / \mathbb{Z}$-form subject to $\mu_{M}$, which resembles to the Brown-Kervaire quadratic forms in the formulation. It has the most similar properties of the Brown-Kervaire quadratic forms, e.g., the isomorphism class of the form is a homotopy invariant if the manifold has vanishing $\mathrm{Wu}$ classes. A bit surprising to us, this invariant applies to give a classification of $(n-2)$-connected $2 n$-dimensional $\pi$-manifolds up to homotopy equivalence and homeomorphism $(n \geq 4)$.

To state our main results, let us start with some notations.

Let $\left\{Y_{k}\right\}_{k \in \mathbb{N}}$ be a connected spectrum with $U \in H^{0}(Y) \cong \mathbb{Z}$ a generator so that $i^{*} U \in H^{0}\left(S^{0}\right)$ a generator, where $i: S^{0} \rightarrow Y$ is the inclusion map of the spectrum.

Definition 1.1. (i) $\left\{Y_{k}\right\}_{k \in \mathbb{N}}$ is called $\Phi$-orientable if $S q^{2} U=0, \chi\left(S q^{n+2}\right)(U)=$ 0 and $0 \in \Phi(U)$, where $\Phi$ is a secondary cohomology operator associated with the Adem relation (see Section 3 for the definition):

$$
\begin{array}{ll}
\chi\left(S q^{n}\right) S q^{3}+\chi\left(S q^{n+2}\right) S q^{1}+S q^{1} \chi\left(S q^{n+2}\right)=0 & n=2(\bmod 4) \\
\chi\left(S q^{n}\right) S q^{3}+S q^{1} \chi\left(S q^{n+2}\right)=0 & n=0(\bmod 4) \\
\chi\left(S q^{n+1}\right) S q^{2}+S q^{1} \chi\left(S q^{n+2}\right)=0 & n=1(\bmod 4)
\end{array}
$$

where $\chi: \mathcal{A}_{2} \rightarrow \mathcal{A}_{2}$ is the anti-automorphism of the Steenrod algebra $\mathcal{A}_{2}$ [1].

A spherical fibration $\xi$ (a manifold) is called $\Phi$-orientable if its Thom spectrum $T \xi$ (stable normal bundle $\nu_{M}$ ) is. We define the universal $\Phi$ orientable $\Omega$-spectrum $\widetilde{W}(n)$ by setting $\widetilde{W}_{k}(n)$ to be the total space of the following Postnikov tower: 


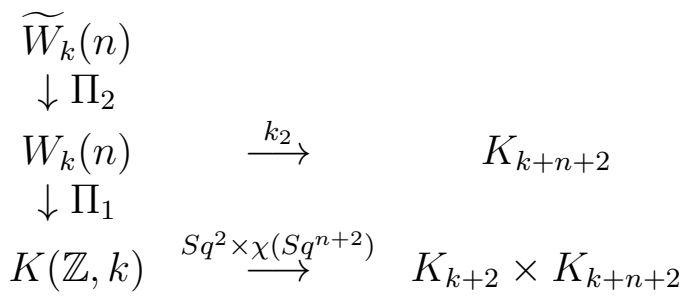

where $K_{i}=K\left(\mathbb{Z}_{2}, i\right), K(\mathbb{Z}, i)$ are the Eilenberg-Maclane spaces, $k_{2} \in$ $\Phi\left(\Pi_{1}{ }^{*} l_{k}\right)$ and $l_{k}$ is the basic class.

Note that a spectrum $Y$ is $\Phi$-orientable if and only if $U \in H^{0}(Y)$ can be lifted to a map $w: Y \rightarrow \widetilde{W}(n)$. We call such a lifting a $\Phi$-orientation of $Y$. A $\Phi$-orientation of a manifold is understood as a $\Phi$-orientation of its Thom spectrum.

Remark 1.2. The sphere spectrum $S^{0}$ is $\Phi$-orientable. Thus stably parallelizable manifolds are $\Phi$-orientable.

Our main results are:

Theorem 1.3. Let $M$ be a $\Phi$-oriented manifold of dimension $2 n$, where $n \neq 3(\bmod 4)$. Then there is a function $\phi_{M}: H^{n-1}\left(M, \mathbb{Z}_{4}\right) \rightarrow \mathbb{Q} / \mathbb{Z}$ such that, for all $x, y \in H^{n-1}\left(M, \mathbb{Z}_{4}\right)$,

$$
\phi_{M}(x+y)=\phi_{M}(x)+\phi_{M}(y)+j\left(x \cup S q^{2} y\right)[M]
$$

where $j: \mathbb{Z}_{2} \rightarrow \mathbb{Q} / \mathbb{Z}$ is the inclusion.

Remark 1.4. In general, $\phi_{M}$ depends on the $\Phi$-orientation, just like the Kervaire quadratic form depends on the framing of the manifold. We will prove that $\phi_{M}(x)$ depends only on the $\Phi$-oriented bordism class $[M, x]$.

Remark 1.5. If $n=3(\bmod 4)$, the analogous definition gives only a linear function.

Let $B \operatorname{Spin}_{G}$ be the classifying space for spherical Spin fibrations. By Brown[4], a $W u$ orientation of a Spin spherical fibration $\xi \searrow M$ is a lifting of the classifying map $\xi: M \rightarrow B \operatorname{Spin}_{G}$ to $B \operatorname{Spin}_{G}\left\langle v_{n+2}\right\rangle$. A Wu orientation of $\nu_{M}$, the stable normal bundle of $M$, is understood as a Wu orientation of $M$, where $B \operatorname{Spin}_{G}\left\langle v_{n+2}\right\rangle \rightarrow B \operatorname{Spin}_{G}$ is a principal fibration with $v_{n+2} \in H^{n+2}\left(B \operatorname{Spin}_{G}, \mathbb{Z}_{2}\right)$ as the $k$-invariant.

We call quadratic forms $\phi_{M_{i}}: H^{n-1}\left(M_{i}, \mathbb{Z}_{4}\right) \rightarrow \mathbb{Q} / \mathbb{Z}, i=1,2$ Witt equiavlent if there exists an isomorphism $\tau: H^{n-1}\left(M_{1}, \mathbb{Z}_{4}\right) \rightarrow$ $H^{n-1}\left(M_{2}, \mathbb{Z}_{4}\right)$ so that $\phi_{M_{2}}(\tau x)=\phi_{M_{1}}(x)$ for all $x \in H^{n-1}\left(M_{1}, \mathbb{Z}_{4}\right)$. 
Theorem 1.6. Let $M_{1}$ and $M_{2}$ be $\Phi$-oriented $2 n$-manifolds. Suppose that the $W u$ classes $v_{n+2-2^{j}}\left(\nu_{M_{i}}\right)=0$ for all $2^{j} \leq n+2$. If $f: M_{1} \rightarrow$ $M_{2}$ is a homotopy equivalence preserving the spin structure (resp. Wu orientation) if $n=0,1(\bmod 4)(\operatorname{resp} . n=2(\bmod 4))$. Then

$$
\phi_{M_{1}}\left(f^{*} x\right)=\phi_{M_{2}}(x)
$$

for all $x \in H^{n-1}\left(M_{2}, \mathbb{Z}_{4}\right)$.

Since the $\mathrm{Wu}$ class $v_{0}=1$, the assumption in the above theorem implies that $n+2 \neq 2^{i}$ for any integer $i$.

For framed manifolds, the Brown-Kervaire secondary quadratic forms have the following property:

Proposition 1.7. If $M$ is a framed manifold of dimension $2 n$, where $n \neq 3(\bmod 4)$. Then $\phi_{M}$ factors through $\mathbb{Z}_{4} \subset \mathbb{Q} / \mathbb{Z}\left(\right.$ resp. $\left.\mathbb{Z}_{2} \subset \mathbb{Q} / \mathbb{Z}\right)$, provided $n=2(\bmod 4)($ resp. $n=0,1(\bmod 4))$.

To state the next results, we need some preliminaries.

Let $H$ be a finitely generated abelian group, and

$$
\mu: \operatorname{Hom}\left(H, \mathbb{Z}_{2}\right) \otimes \operatorname{Hom}\left(H, \mathbb{Z}_{2}\right) \rightarrow \mathbb{Z}_{2}
$$

be a symmetric bilinear form. We say that $\mu$ is of diagonal zero if $\mu(x, x)=0$ for each $x \in \operatorname{Hom}\left(H, \mathbb{Z}_{2}\right)$. A function $\phi: \overline{\operatorname{Hom}\left(H, \mathbb{Z}_{4}\right)} \rightarrow$ $\mathbb{Q} / \mathbb{Z}$ is called quadratic with respect to $\mu$ if

$$
\phi(x+y)=\phi(x)+\phi(y)+j(\mu(x, y))
$$

where $j: \mathbb{Z}_{2} \rightarrow \mathbb{Q} / \mathbb{Z}$ is the inclusion. This gives a triple $(H, \mu, \phi)$. We say triples $\left(H_{1}, \mu_{1}, \phi_{1}\right)$, and $\left(H_{2}, \mu_{2}, \phi_{2}\right)$ are isometric if there exists an isomorphism $\tau: H_{1} \rightarrow H_{2}$ such that $\mu_{1}(x, y)=\mu_{2}(\tau x, \tau y)$ and $\phi_{1}(x)=\phi_{2}(\tau x)$ for all $x, y$. We denote by $[H, \mu, \phi]$ the isometry class of a triple.

Remark 1.8. Since the natural map $\operatorname{Hom}\left(H_{n-1}(M), \mathbb{Z}_{2}\right) \rightarrow H^{n-1}\left(M, \mathbb{Z}_{2}\right)$ is not an isomorphism in general, the notions of isometry associated with $\mu$ and $\mu_{M}$ as above are different. They do agree however for $(n-2)$ connected manifolds which we will assume in the later application. We will use both of them when necessary.

Let $i$ denote the maximal exponent of the 2-torsion subgroup of $H_{n-1}(M)$ and let $S q_{i}^{1} \in H^{n}\left(K\left(\mathbb{Z}_{2^{i}}, n-1\right), \mathbb{Z}_{2}\right) \cong \mathbb{Z}_{2}$ be the unique generator. Considering $S q_{i}^{1}$ as a cohomology operation we get a function

$$
q_{M}\left(S q_{i}^{1}\right): H^{n-1}\left(M, \mathbb{Z}_{2^{i}}\right) \rightarrow \mathbb{Z}_{2}
$$

This gives a homomorphism since $S q_{i}^{1} x \cup S q_{i}^{1} y=S q_{i}^{1}\left(x \cup S q_{i}^{1} y\right)=0$ for $x, y \in H^{n-1}\left(M, \mathbb{Z}_{2}\right)$. We denote by $\left[H_{n-1}(M), \mu_{M}, q_{M}\left(S q_{i}^{1}\right)\right]$ for the 
isometry class of the triple. By [6], the Kervaire invariant of a smooth framed manifold of dimension $2 n$, where $n \neq 2^{i}-1$, is zero. For $i \leq 5$, there are smooth manifolds of dimension $2^{i+1}-2$ of Kervaire invariant 1. It is still an open problem whether there is such a manifold for $i \geq 6$.

The Kervaire invariant does not depend on the framings of the underlying $2 n$-manifold if $n \neq 1,3,7$ and the manifold is highly connected, e.g. $(n-2)$-connected. Moreover, by [4] the Kervaire form is a homotopy invariant if $n \neq 1,3,7$ and $(n-2)$-connected.

Let $M$ be a $(n-2)$-connected $2 n$-dimensional $\pi$-manifold. Observe that if $n \geq 3$, there exists a $(n-2)$-connected $\pi$-manifold, $N$, so that $M=N \# X$ and $H_{n}(N, \mathbb{Q})=0$, where $X$ is a $(n-1)$-connected $2 n$ manifold. Since the classification of $(n-1)$-connected $2 n$-manifolds is well understood [25], for convenience in the following theorem we assume that $H_{n}(M, \mathbb{Q})=0$. For such a manifold, consider the correspondence

$$
\pi: M \longmapsto\left[H_{n-1}(M), \mu_{M}, \phi_{M}\right]\left(\operatorname{resp.}\left[H_{n-1}(M), \mu_{M}, \phi_{M}, q_{M}\left(S q_{2^{i}}^{1}\right)\right]\right)
$$

if $n=0(\bmod 2)($ resp. $n=1(\bmod 2))$.

In the following theorem let $\alpha(n+2)$ be the number of $1^{\prime} s$ in the binary expansion of $n+2$.

Theorem 1.9. Suppose $n \geq 4$ and $\alpha(n+2) \geq 2$. Then $\pi$ gives a 1-1 correspodence between the homeomorphism types (resp. homotopy types) of $(n-2)$-connected $2 n$-dimensional $\pi$-manifolds $M$ so that $H_{n}(M, \mathbb{Q})=0$ with the following algebraic data

(a) $\wp_{n}=\left\{[H, \mu, \phi]: \operatorname{diag} \mu=0\right.$ and $\phi$ factors through $\left.j: \mathbb{Z}_{4} \rightarrow \mathbb{Q} / \mathbb{Z}\right\}$ if $n=2(\bmod 4)$,

(b) $\wp_{n}=\left\{[H, \mu, \phi]: \phi\right.$ factors through $\left.j: \mathbb{Z}_{2} \rightarrow \mathbb{Q} / \mathbb{Z}\right\}$ if $n=0(\bmod 4)$,

(c) $\wp_{n}=\left\{[H, \mu, \phi, \omega]: \omega \in \operatorname{Hom}\left(\operatorname{tor}(H) \otimes \mathbb{Z}_{2^{i}}, \mathbb{Z}_{2}\right), \phi\right.$ factors through $\left.j: \mathbb{Z}_{2} \rightarrow \mathbb{Q} / \mathbb{Z}\right\}$ if $n=1(\bmod 4)$,

(d) $\wp_{n}=\left\{[H, \mu, \omega]: \omega \in \operatorname{Hom}\left(\right.\right.$ tor $\left.\left.H \otimes \mathbb{Z}_{2^{i}}, \mathbb{Z}_{2}\right)\right\}$ if $n=3(\bmod 4)$.

where $i$ is the highest exponent of the 2-cyclic subgroup of $H$ and if $n=1(\bmod 2)$, the pairing $\mu(x, x)=0($ resp. $\delta \omega(x))$ if $x$ can be lifted to $a \mathbb{Z}_{4}$ class with order 4 (resp. $x$ is of order 2 ), $\delta \in\{0,1\}$ is ambiguous.

Remark 1.10. The classification of $(n-2)$-connected $2 n$-manifolds with torsion free homology groups has been given by Ishimoto[9][10]. But his method does not work if the homology group has torsion.

The organization of this paper is as follows.

In $\S 2$ we define the secondary Brown-Kervaire form and state its basic properties.

In $\S 3$, we set up the necessary foundations on the stable homotopy theory of the Eilenberg-Maclane spaces. 
In $\S$, we are addressed to show Theorems 1.3 and 1.6.

In $\S 5$, we prove Theorem 1.9 .

\section{A $\mathbb{Q} / \mathbb{Z}$-QUADRATIC FORM OF $\Phi$-ORIENTED MANIFOLDS}

Let us begin with some conventions. All homology/cohomology groups will be with integral coefficients unless otherwise stated. All spaces will have base points. Let

(i) $[X, Y]$ denote the set of homotopy classes of pointed maps from $X$ to $Y$.

(ii) $\{X, Y\}=\lim \left[S^{k} X, S^{k} Y\right]$.

(iii) $\pi_{*}^{s}(X)$ be its 2-localization to simplify the notation.

Let $\kappa: K\left(\mathbb{Z}_{4}, n-1\right) \times K\left(\mathbb{Z}_{4}, n-1\right) \rightarrow K\left(\mathbb{Z}_{4}, n-1\right)$ be the multiplication of $K\left(\mathbb{Z}_{4}, n-1\right)$ and let $H(\kappa)$ be the Hopf construction of $\kappa$.

Proposition 2.1. The homomorphism

$$
H(\kappa)_{*}: \pi_{2 n}^{s}\left(K\left(\mathbb{Z}_{4}, n-1\right) \wedge K\left(\mathbb{Z}_{4}, n-1\right)\right) \rightarrow \pi_{2 n}^{s}\left(K\left(\mathbb{Z}_{4}, n-1\right)\right)
$$

is injective if $n \neq 3(\bmod 4)$, and zero if $n=3(\bmod 4)$.

Remark 2.2. If $\mathbb{Z}_{4}$ is replaced by $\mathbb{Z}_{2}$, then $H(\kappa)_{*}$ is trivial.

By Theorem 3.1 and the proof of it, we obtain

$$
\begin{aligned}
& \pi_{2 n}^{s}\left(K\left(\mathbb{Z}_{4}, n-1\right) \wedge K\left(\mathbb{Z}_{4}, n-1\right)\right) \cong \mathbb{Z}_{2} \text { if } n \geq 4 \\
& \pi_{2 n}^{s}\left(K\left(\mathbb{Z}_{4}, n-1\right)\right) \cong \mathbb{Z}_{4} \text { if } n=2(\bmod 4)
\end{aligned}
$$

Let $\lambda_{0}$ be a generator of $\operatorname{Im} H(\kappa)_{*}$ if $n \neq 2(\bmod 4)$, and a specified generator of $\pi_{2 n}^{s}\left(K\left(\mathbb{Z}_{4}, n-1\right)\right) \cong \mathbb{Z}_{4}$ otherwise. For a given spectrum $Y$, let

$$
H_{*}\left(K\left(\mathbb{Z}_{4}, n-1\right) ; Y\right)=\lim \pi_{*+k}\left(K\left(\mathbb{Z}_{4}, n-1\right) \wedge Y_{k}\right) .
$$

Theorem 2.3. Suppose that $\left\{Y_{k}\right\}_{k \in \mathbb{N}}$ is a $\Phi$-orientable spectrum. Then there exists a homomorphism

$$
h: H_{2 n}\left(K\left(\mathbb{Z}_{4}, n-1\right) ; Y\right) \rightarrow \mathbb{Q} / \mathbb{Z}
$$

such that $h(\lambda)=\frac{1}{4}\left(\right.$ resp. $\left.\frac{1}{2}\right)$ if $n=2(\bmod 4)(\operatorname{resp} . n=0,1(\bmod 4))$, where $\lambda=i_{*}\left(\lambda_{0}\right)$ and $i_{*}: H_{2 n}\left(K\left(\mathbb{Z}_{4}, n-1\right) ; S^{0}\right) \rightarrow H_{2 n}\left(K\left(\mathbb{Z}_{4}, n-1\right) ; Y\right)$ is induced by the inclusion. 
SECONDARY BROWN-KERVAIRE QUADRATIC FORMS AND $\pi$-MANIFOLDS 7

Definition 2.4. A Poincaré triple $(M, \xi, \alpha)$ of dimension $2 n$ consists of

(i) A CW complex $M$ with finitely generated homology.

(ii) A fibration $\xi$ over $M$ with fiber homotopy equivalent to $S^{k-1}, k$ large.

(iii) $\alpha \in \pi_{2 n+k}(T \xi)$ such that an $(2 n+k)$ Spanier-Whitehead S-duality is given by

$$
S^{2 n+k} \stackrel{\alpha}{\longrightarrow} T \xi \stackrel{\Delta}{\longrightarrow} T \xi \wedge M^{+}
$$

where $T \xi$ is the Thom complex of $\xi$ and $\Delta$ is the diagonal map.

Let $A_{\alpha}:\left\{M_{+}, K\left(\mathbb{Z}_{4}, n-1\right)\right\} \rightarrow\left\{S^{2 n+k}, T \xi \wedge K\left(\mathbb{Z}_{4}, n-1\right)\right\}$ be the $S$-duality map.

Definition 2.5. Let $(M, \xi, \alpha)$ be a Poincaré triple and $w$ is a $\Phi$-orientation of the Thom spectrum $T \xi$. For a homomorphism $h$ in Theorem 2.3, let

$$
\phi_{w, h}: H^{n-1}\left(M, \mathbb{Z}_{4}\right) \rightarrow \mathbb{Q} / \mathbb{Z}
$$

be defined by setting

$$
\phi_{w, h}(x)=h\left(\left[(w \wedge i d) A_{\alpha}(x)\right]\right) .
$$

Theorem 2.6. Let $\phi_{w, h}$ be defined as above. Then for all $x, y \in H^{n-1}\left(M, \mathbb{Z}_{4}\right)$, (i) If $n \neq 3(\bmod 4)$, the function is quadratic, i.e.

$$
\phi_{w, h}(x+y)=\phi_{w, h}(x)+\phi_{w, h}(y)+j\left(x \cup S q^{2} y\right)[M]
$$

where $j: \mathbb{Z}_{2} \rightarrow \mathbb{Q} / \mathbb{Z}$ is the inclusion;

(ii) If $n=3(\bmod 4), \phi_{w, h}$ is linear, i.e.

$$
\phi_{w, h}(x+y)=\phi_{w, h}(x)+\phi_{w, h}(y) .
$$

Now we want to study how the function $\phi_{w, h}$ depends on the choice of the orientation of the Thom spectrum $T \xi$.

Let $w_{i}, i=1,2$ are orientations of the Thom spectrum $T \xi$. Let

$$
d_{1}\left(w_{1}, w_{2}\right) \in H^{1}(T \xi) \oplus H^{n+1}(T \xi)
$$

denote the difference of the composition maps $\Pi_{2} w_{1}$ and $\Pi_{2} w_{2}$, where $\Pi_{2}$ is as in the definition of the universal $\Omega$-spectrum $\widetilde{W}(n)$. Clearly, $w_{1}$ and $w_{2}$ are homotopy if and only if $d_{1}\left(w_{1}, w_{2}\right)=0$ and a secondary obstruction vanishes. The following theorem shows that the secondary obstruction does not affect our quadratic function $\phi_{w, h}$.

Theorem 2.7. Let $\phi_{w_{i}, h}$ be the quadratic forms associated with $\left(w_{i}, h\right)$, $i=1,2$. If $d_{1}\left(w_{1}, w_{2}\right)=0$, then $\phi_{w_{1}, h}(x)=\phi_{w_{2}, h}(x)$ for all $x \in$ $H^{n-1}\left(M, \mathbb{Z}_{4}\right)$. 
In general, the quadratic form $\phi_{w, h}$ does depend on the choice of $w$ and $h$. In order to obtain a well-defined invariant of the $\Phi$-oriented manifold, we now choose certain type of $\Phi$-orientations of the Thom spectrum $T \xi$ in an universal way and then define the Brown-Kervaire secondary quadratic forms to be the quadratic functions associated to those $\Phi$-orientations.

Let $\gamma \searrow \operatorname{BSpin}_{G}$ be the universal Spin spherical fibration and $U \in$ $H^{0}\left(M \operatorname{Spin}_{G}, \mathbb{Z}_{2}\right)$ the universal Thom class. Note that

$$
\begin{aligned}
& \chi\left(S q^{n+2}\right) U=\chi\left(S q^{n+1}\right) S q^{1} U=0 \text { if } n \text { is odd } \\
& \chi\left(S q^{n+2}\right) U=\chi\left(S q^{n}\right) S q^{2} U=0 \text { if } n=0(\bmod 4),
\end{aligned}
$$

Thus $U$ lifts to a map $f: M \operatorname{Spin}_{G} \rightarrow W(n)$. By the Thom isomorphism, $f^{*} k_{2}$ gives an element of $\overline{k_{2}} \in H^{n+2}\left(B \operatorname{Spin}_{G}, \mathbb{Z}_{2}\right)$. Let $\pi: B \operatorname{Spin}_{G}\left\langle\bar{k}_{2}\right\rangle \rightarrow B \operatorname{Spin}_{G}$ be the principal fibration with $k$-invariant $\overline{k_{2}}$.

If $n=2(\bmod 4)$, we get a similar principal fibration $\pi: B \operatorname{Spin}_{G}\left\langle\overline{k_{2}}\right\rangle \rightarrow$ $\operatorname{BSpin}_{G}\left\langle v_{n+2}\right\rangle$, where $B \operatorname{Spin}_{G}\left\langle v_{n+2}\right\rangle \rightarrow B \operatorname{Spin}_{G}$ is the fibration with fibre $K_{n+1}$ and $k$-invariant $v_{n+2}$.

It is easy to see that the fibration $\pi^{*} \gamma$ is $\Phi$-orientable. Clearly the classifying map of every $\Phi$-orientable stable spherical fibration lifts to $\operatorname{BSpin}_{G}\left\langle\overline{k_{2}}\right\rangle$.

Definition 2.8. The fibration $\pi^{*} \gamma$ is called the universal $\Phi$-orientable spherical Spin fibration. Its Thom spectrum, $\operatorname{MSpin}_{G}\left\langle\overline{k_{2}}\right\rangle$, is called the universal $\Phi$-orientable Thom spectrum.

For a closed $\Phi$-orientable manifold $M^{2 n}$, there is a Poincaré triple $\left(M, \nu_{M}, \alpha\right)$ where $\nu_{M}$ is the stable normal bundle and $\alpha \in \pi_{2 n+k}\left(T \nu_{M}\right)$ is the normal invariant of $M$ (obtained by the Thom-Pontryagin construction.)

Definition 2.9. Fix a connected spectral map $\mathbf{u}: M \operatorname{Spin}_{G}\left\langle\overline{k_{2}}\right\rangle \rightarrow$ $\widetilde{W}(n)$ and a homomorphism $h$ in Theorem 2.3. For a $\Phi$-orientable manifold $M$, let

$$
\phi_{M}=\phi_{w, h}
$$

where $w=\mathbf{u} \circ T(v)$ and $T(v)$ is the Thom map of a classifying bundle map of the stable bundle $\nu_{M}$.

Now we prove Theorem 1.6 assuming Theorem 2.7.

Proof of Theorem 1.0. Let $\xi_{i}=\nu_{M_{i}}$ be the stable normal bundle of $M_{i}$ and $\alpha_{i} \in \pi_{2 n+k}\left(T \xi_{i}\right)$ be the normal invariant, $i=1,2$. By the definition, $\phi_{M_{i}}=\phi_{w_{i}, h}$ where $w_{i}=\mathbf{u} \circ T\left(v_{i}\right)$ and $T\left(v_{i}\right): T\left(\xi_{i}\right) \rightarrow M \operatorname{Spin}_{G}\left\langle\bar{k}_{2}\right\rangle$ the Thom map. 
Let $\tilde{f}: f^{*} \xi_{2} \rightarrow \xi_{2}$ be a bundle map over the homotopy equivalence $f$. Let $\alpha_{3}=T(\tilde{f})_{*}^{-1} \alpha_{2}$, where $T(\tilde{f})$ is the Thom map of $\tilde{f}$. The Poincaré triple $\left(M_{1}, f^{*} \xi_{2}, \alpha_{3}\right)$ together with the $\Phi$-orientation $w_{2} \circ T(\tilde{f})$ gives a quadratic form $\phi_{3}$, where $w_{2}=\mathbf{u} \circ T\left(v_{2}\right)$ is a $\Phi$-orientation of $M_{2}$. By 2.5 we get that

$$
\phi_{3}\left(f^{*} x\right)=\phi_{M_{2}}(x)
$$

for all $x \in H^{n-1}\left(M_{2}, \mathbb{Z}_{4}\right)$.

To prove the desired result, it suffices to prove $\phi_{3}=\phi_{M_{1}}$.

Note that $f^{*} \xi_{2}$ and $\xi_{1}$ are stably equivalent as spherical fibration since $f$ is a homotopy equivalence. Thus we can regard $f^{*} \xi_{2}$ and $\xi_{1}$ as the the same and so get two orientations for $\xi_{1},\left(\mathbf{u} \circ T\left(v_{1}\right), h\right)$ and $(\mathbf{u} \circ$ $\left.T\left(v_{2}\right) \circ T(\tilde{f}), h\right)$. Since $f$ preserves the Spin structures/Wu orientations, $\pi \circ v_{2} \circ f \simeq \pi \circ v_{1}$, where $\pi: B \operatorname{Spin}_{G}\left\langle\overline{k_{2}}\right\rangle \rightarrow \operatorname{BSpin}_{G} / B \operatorname{Spin}_{G}\left\langle v_{n+2}\right\rangle$ is the principal fibration as above. This clearly implies that there exists a fibre automorphism $g \in A u t\left(\xi_{1}\right)$ over the identity such that

$$
T\left(\pi \circ v_{2} \circ \tilde{f}\right) \simeq T\left(\pi \circ v_{1}\right) \circ T(g) .
$$

Notice that $g$ gives a unique element $g_{0} \in\left[M_{1}, G_{k}\right]$, where $G_{k}$ is the space of self homotopy equivalences of $S^{k}$. By a formula in Brown [4], the $(n+1)$-dimensional component of $d_{1}\left(\mathbf{u} \circ T\left(v_{1}\right) \circ T(g), \mathbf{u} \circ\right.$ $\left.T\left(v_{1}\right)\right)$ is $\sum v_{n+2-2^{i}} \cup g_{0}^{*} u_{2^{i}-1}$, where $u_{2^{i}-1}$ is the transgression of $w_{2^{i}} \in$ $H^{2 i}\left(B G_{k}, Z_{2}\right)$. By assumption, it must vanish since the $\mathrm{Wu}$ classes vanish. On the other hand, the 1-dimensional component of $d_{1}\left(\mathbf{u} \circ T\left(v_{1}\right) \circ\right.$ $\left.T(g), \mathbf{u} \circ T\left(v_{1}\right)\right)$ is determined by the Spin structures and so it vanishes since $f$ preserves the Spin structures. By Theorem 2.7 it follows that

$$
\phi_{M_{1}}=\phi_{4},
$$

the quadratic form associated with the Poincaré triple $\left(M_{1}, \xi_{1}, \alpha_{1}\right)$ and the $\Phi$-orientation $w_{2} \circ T(\tilde{f})$.

Note that in the definitions of $\phi_{3}$ and $\phi_{4}$ the only different ingradients are the normal invariants, after identifying $\xi_{1}$ with $f^{*} \xi_{2}$. By Theorem 2.7 once again $\phi_{3}=\phi_{4}$. This implies the desired result.

Now we prove Proposition 1.7.

Proof of Proposition 1.7. Since $M$ is a framed manifold, the stable normal bundle is trivial, i.e. the classifying map of $\nu_{M}$ factors through a point. Choose a $\Phi$-orientation $w=\mathbf{u} \circ T(v): \nu_{M}$ with $v$ the bundle map of $\nu_{M}$ to the trivial $k$-bundle on a point, then $\phi_{M}(x)$ factors through the stable homotopy group $\pi_{2 n}^{s}\left(K\left(\mathbb{Z}_{4}, n-1\right)\right)$. By Theorem $3.1 \pi_{2 n}^{s}\left(K\left(\mathbb{Z}_{4}, n-1\right)\right) \cong \mathbb{Z}_{4}$ if $n=2(\bmod 4)$ and the order of elements in $\pi_{2 n}^{s}\left(K\left(\mathbb{Z}_{4}, n-1\right)\right.$ is at most 2 if $n=0,1(\bmod 4)$. On the other hand, by Theorem 1.6 the definition of $\phi_{M}$ does not depend on the choice of 
the $\Phi$-orientations since $M$ is a framed manifold. This completes the proof.

\section{Some PRELIMINARIES ON STABLE HOMOTOPY THEORY}

In this section we calculate the stable homotopy groups $\pi_{2 n}^{s}(K(\pi, n-$ 1)) (see Theorem 3.1). We will also introduce some 2-stage Postnikov tower which will give the secondary cohomology operation $\Phi$ used in Section 81 .

Theorem 3.1. The $2 n$-th stable homotopy group of $K(\pi, n-1)$ for $n \geq 4$ is as follows:

\begin{tabular}{|r|c|c|c|c|}
\hline \hline$n \geq 4$ & $0(\bmod 4)$ & $1(\bmod 4)$ & $2(\bmod 4)$ & $3(\bmod 4)$ \\
\hline$\pi_{2 n}^{s}(K(\pi, n-1))$ & $\left(\mathbb{Z}_{2}\right)^{2(t+k)+s+p}$ & $\left(\mathbb{Z}_{2}\right)^{t+2 k+s+p}$ & $\left(\mathbb{Z}_{4}\right)^{t+k} \oplus\left(\mathbb{Z}_{2}\right)^{s+p}$ & $\left(\mathbb{Z}_{2}\right)^{k+s+p}$ \\
\hline
\end{tabular}

where $p=\left(\begin{array}{c}t+k+s \\ 2\end{array}\right)$ and $\pi=G_{0} \times \mathbb{Z}^{t} \times \mathbb{Z}_{2^{i_{1}}} \times \cdots \times \mathbb{Z}_{2^{i_{k}}} \times \mathbb{Z}_{2}^{s}, i_{j} \geq 2$ if $1 \leq j \leq k$ and $G_{0} \otimes \mathbb{Z}_{2}=0$.

When $\pi=\mathbb{Z}$, Theorem 3.1 follows from [17].

Proof. It is easy to know that(since we are computing the 2-localization)

$$
\pi_{2 n}^{s}(K(\pi, n-1))=\pi_{2 n}^{s}\left(K\left(\pi / G_{0}, n-1\right)\right)
$$

Assume $G_{0}=0$ from now on. If $\pi=\pi_{1} \bigoplus \pi_{2}$ with $\pi_{1}$ nontrivial and $\pi_{2}$ a nontrivial cyclic group, then

$$
K(\pi, n-1)=K\left(\pi_{1}, n-1\right) \times K\left(\pi_{2}, n-1\right)
$$

and we have by a result in $[3]$ that

$$
\begin{gathered}
\pi_{2 n}^{s}(K(\pi, n-1))=\bigoplus_{i=1,2} \pi_{2 n}^{s}\left(K\left(\pi_{i}, n-1\right)\right) \bigoplus \pi_{2 n}^{s}\left(K\left(\pi_{1}, n-1\right) \wedge K\left(\pi_{2}, n-1\right)\right) \\
=\bigoplus_{i=1,2} \pi_{2 n}^{s}\left(K\left(\pi_{i}, n-1\right)\right) \bigoplus H_{n+1}\left(K\left(\pi_{1}, n-1\right), \pi_{2}\right)
\end{gathered}
$$

An easy calculation shows that $H_{n+1}\left(K\left(G_{1}, n-1\right), G_{2}\right)=Z_{2}$ if $G_{1}, G_{2}$ are nontrivial cyclic groups and thus $H_{n+1}\left(K\left(\pi_{1}, n-1\right), \pi_{2}\right)=Z_{2}^{t+k+s-1}$.

On the other hand we know groups $\pi_{2 n}^{s}(K(\mathbb{Z}, n-1))$ and $\pi_{2 n}^{s}\left(K\left(\mathbb{Z}_{2}, n-\right.\right.$ 1)) by results in [20], [17]. To complete the proof it remains to calculate $\pi_{2 n}^{s}\left(K\left(\mathbb{Z}_{2^{i}}, n-1\right)\right)$ for $2 \leq i<\infty$ which will be given in the following results.

Recall that for each locally finite connected CW complex $X$ we can define a space

$\Gamma_{q} X=S^{q-1} \propto_{T} X \wedge X=S^{q-1} \times(X \wedge X) /\{(x, y, z) \sim(-x, z, y) ;(x, *) \sim *\}$ 
for every $q \in Z_{+}$. By 20 Theorem 1.11, for a $(n-2)$-connected space $X, \Gamma_{q} X$ is $(2 n-3)$-connected. Moreover, if $X=K(\pi, n-1)$, we have a fibration

$$
G_{q} \rightarrow \Sigma^{q} K(\pi, n-1) \rightarrow K(\pi, q+n-1)
$$

where $G_{q} \simeq \Sigma^{q} \Gamma_{q}(K(\pi, n-1))$ through dimension $(3 n+q-3)$. Thus $\pi_{i}^{s}(K(\pi, n-1)) \cong \pi_{i}^{s}\left(\Gamma_{q}(K(\pi, n-1))\right.$ for $n<i<3 n-3$.

When $q=1, \Gamma_{q} X=X \wedge X$. The corresponding sequence is :

$$
\Sigma F_{n-1}(\pi) \stackrel{H(\kappa)}{\rightarrow} \Sigma K(\pi, n-1) \rightarrow K(\pi, n)
$$

where $F_{n-1}(\pi)=K(\pi, n-1) \wedge K(\pi, n-1)$. After $q-1$ time suspensions we get a fibration sequence at least in dimensions less than $3 n+q-4$

$$
\Sigma^{q} F_{n-1}(\pi) \stackrel{\Sigma^{q-1} H(\kappa)}{\rightarrow} \Sigma^{q} K(\pi, n-1) \rightarrow \Sigma^{q-1} K(\pi, n)
$$

Let $q$ be large enough so that we are always in the stable range and let $r=q+2 n$, then we have an exact sequence

$$
\begin{gathered}
\cdots \rightarrow \pi_{2 n+2}^{s}(K(\pi, n)) \stackrel{\partial}{\rightarrow} \pi_{r}\left(\Sigma^{q} F_{n-1}(\pi)\right) \rightarrow \\
\rightarrow \pi_{2 n}^{s}(K(\pi, n-1)) \rightarrow \pi_{2 n+1}^{s}(K(\pi, n)) \stackrel{\partial}{\rightarrow} \cdots
\end{gathered}
$$

Since we know that $\pi_{r}\left(\Sigma^{q} F_{n-1}(\pi)\right)=\mathbb{Z}_{2}$ for $\pi=\mathbb{Z}$ and $\mathbb{Z}_{2^{i}}$, we can determine inductively $\pi_{2 n}^{s}(K(\pi, n-1))$ up to extension if we know the map $\partial$.

Lemma 3.2. For $\pi=\mathbb{Z}$ or $\mathbb{Z}_{2^{i}}$, a homotopy class $[g] \in \pi_{2 n+2}^{s}(K(\pi, n))$ has $\partial g \neq 0$ iff $g^{*}\left(\Sigma^{q-1}\left(\iota \cup S q^{2} \iota\right)\right) \neq 0$ where $g: S^{r+1} \rightarrow \Sigma^{q-1} K(\pi, n), \iota \in$ $H^{n}\left(K(\pi, n), \mathbb{Z}_{2}\right)$ is the generator and $g^{*}: H^{*}\left(\Sigma^{q-1} K(\pi, n), \mathbb{Z}_{2}\right) \rightarrow$ $H^{*}\left(S^{r+1}, \mathbb{Z}_{2}\right)$

The proof is similar to that of Lemma 1.3 in [17]. The key points are the followings:

- $g^{*}$ can be nonzero only on element $\Sigma^{q-1}\left(\iota \cup S q^{2} \iota\right)$

- the Hurewicz homomorphism $H: \mathbb{Z}_{2} \cong \pi_{r}\left(\Sigma^{q} F_{n-1}(\pi)\right) \rightarrow H_{r}\left(\Sigma^{q} F_{n-1}(\pi)\right)$ is nonzero

The first statement is clear while the second is an easy consequence of the Whitehead exact sequence(c.f. [26], page 555)

With the lemma above we can now prove the Proposition2.1.

Proof of Proposition 2.1. It suffices to prove that $\partial$ is trivial if $n=$ $0,1,2(\bmod 4)$ and nontrivial if $n=3(\bmod 4)$.

For $n=0,1,2(\bmod 4)$ there is no $g: S^{r+1} \rightarrow \Sigma^{q-1} K(\mathbb{Z}, n)$ such that $g^{*}\left(\Sigma^{q-1}\left(\iota \cup S q^{2} \iota\right)\right) \neq 0$ since $\Sigma^{q-1}\left(\iota \cup S q^{2} \iota\right)$ is detected by the secondary cohomology operation $\varphi_{n}$ in 18 . Thus there is no $g: S^{r+1} \rightarrow$ $\Sigma^{q-1} K\left(Z_{2^{i}}, n\right)$ such that $g^{*}\left(\Sigma^{q-1}\left(\iota \cup S q^{2} \iota\right)\right) \neq 0$ by the naturality of 
secondary cohomology operation and the fact that $\rho_{2^{i}}: K(\mathbb{Z}, n) \rightarrow$ $K\left(\mathbb{Z}_{2^{i}}, n\right)$ corresponding to mod $2^{i}$ reduction induces a homomorphism sending $\Sigma^{q-1}\left(\iota \cup S q^{2} \iota\right)$ to the corresponding element. It follows that $\partial=0$

When $n=3(\bmod 4)$, there is a map $g: S^{r+1} \rightarrow \Sigma^{q-1} K(\mathbb{Z}, n)$ such that $g^{*}\left(\Sigma^{q-1}\left(\iota \cup S q^{2} \iota\right)\right) \neq 0$ since otherwise $\pi_{2 n}^{s}(K(\mathbb{Z}, n-1)) \neq 0$. By the above fact on map $\rho_{2^{i}}$ it is easy to see that there is a map $h: S^{r+1} \rightarrow \Sigma^{q-1} K\left(\mathbb{Z}_{2^{i}}, n\right)$ such that $h^{*}\left(\Sigma^{q-1}\left(\iota \cup S q^{2} \iota\right)\right) \neq 0$.It follows from the lemma above that $\partial h \neq 0$.

With the help of Proposition2.1 and the known results about $\pi_{2 n+j}^{s}(K(\pi, n))$ for $j=0,1$, we can now determine the group $\pi_{2 n}^{s}\left(K\left(\mathbb{Z}_{2^{i}}, n-1\right)\right)$.

Assume $i \geq 2$ in the following unless otherwise stated.

Proposition 3.3. If $n=0(\bmod 2)$, then

$$
\rho_{2^{i}{ }_{*}}: \pi_{2 n}^{s}(K(\mathbb{Z}, n-1)) \rightarrow \pi_{2 n}^{s}\left(K\left(\mathbb{Z}_{2^{i}}, n-1\right)\right)
$$

is an isomorphism.

Before the proof of the Proposition, let's give two remarks which are clear from the proof of the Proposition.

Remark 3.4. If $i=1, \rho_{2^{i} *}$ is onto.

Remark 3.5. If $n=0(\bmod 4)$, then the spherical cohomology class in $\pi_{2 n}^{s}(K(\mathbb{Z}, n-1))$ does not belongs to the image of the natural map: $\pi_{r}\left(\Sigma^{q} F_{n-1}(\mathbb{Z})\right) \rightarrow \pi_{2 n}^{s}(K(\mathbb{Z}, n-1))$.

Proof. Note that we have a commutative diagram

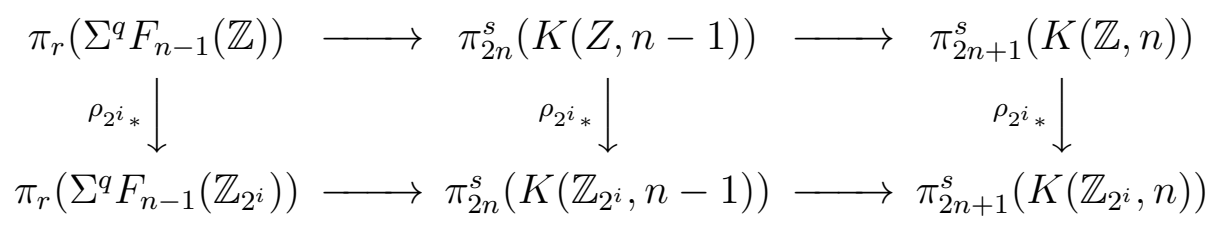

In the above diagram,the two left horizontal maps are injective by Lemma3.2, the left vertical map is obviously an isomorphism while the fact that the right vertical one is also an isomorphism follows by comparing the Whitehead exact sequences of $\Gamma_{q-1}(K(\mathbb{Z}, n))$ and $\Gamma_{q-1}\left(K\left(\mathbb{Z}_{2^{i}}, n\right)\right)$.On the other hand , the fact that the right horizontal map on the bottom line is onto follows from the long exact sequence and the known results about $\pi_{2 n+j}^{s}\left(K\left(\mathbb{Z}_{2^{i}}, n\right)\right)$ for $j=0,1$.

Proposition 3.6. For $n=1(\bmod 2), \pi_{2 n}^{s}\left(K\left(\mathbb{Z}_{2^{i}}, n-1\right)\right)=\pi_{2 n}^{s}(K(\mathbb{Z}, n-$ 1)) $\bigoplus \mathbb{Z}_{2}$. 
SECONDARY BROWN-KERVAIRE QUADRATIC FORMS AND $\pi$-MANIFOLDS13

Proof. The relevant commutative diagram in this case is

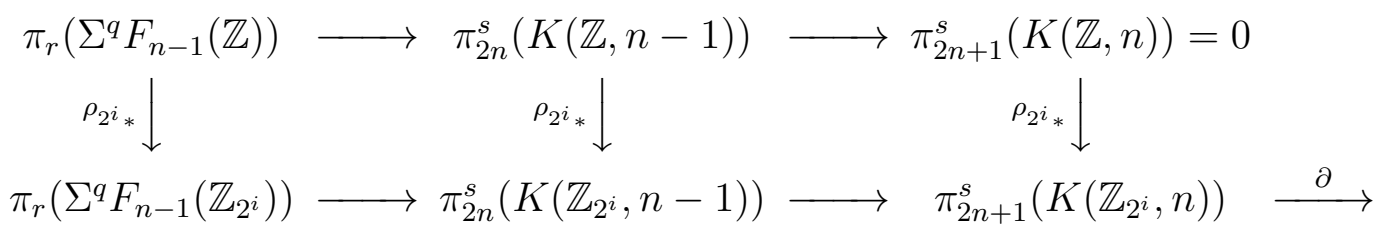

By the same argument as in the last Proposition, we know the map $\partial$ is onto. If $n=3(\bmod 4)$, the two left horizontal maps are trivial by Lemmaß3.2, thus $\pi_{2 n}^{s}\left(K\left(\mathbb{Z}_{2^{i}}, n-1\right)\right) \cong$ coker $\partial \cong \mathbb{Z}_{2}$.

If $n=1(\bmod 4)$, what we can get is an exact sequence

$$
0 \rightarrow \pi_{2 n}^{s}(K(\mathbb{Z}, n-1)) \rightarrow \pi_{2 n}^{s}\left(K\left(\mathbb{Z}_{2^{i}}, n-1\right)\right) \rightarrow \mathbb{Z}_{2} \rightarrow 0
$$

To complete the proof, it suffices to prove the last map in the above sequence has a section.

To do this we need another diagram

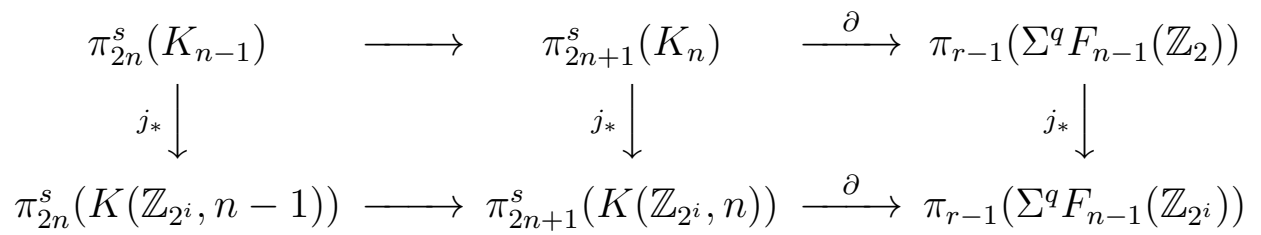

where $j: \mathbb{Z}_{2} \rightarrow \mathbb{Z}_{2^{i}}$ is the natural inclusion.

The same argument as above combined with the proof of Theorem $10.9 \mathrm{in}\left[20\right.$ shows that the two $\partial$ 's are onto and $j_{*}$ induces an isomorphism between kernels of two $\partial$ 's. Finally we get the following diagram which gives the desired section.

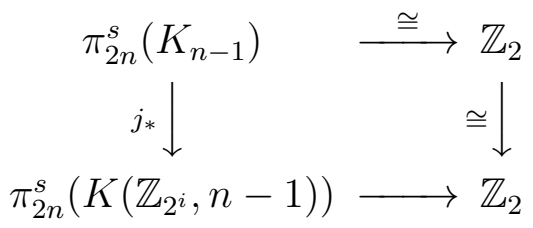

Lemma 3.7. If $n$ is odd and $S q_{i}^{1} \in H^{n}\left(K\left(\mathbb{Z}_{2^{i}}, n-1\right), \mathbb{Z}_{2}\right)$ is a generator. Then

$$
\left(S q_{i}^{1}\right)_{*}: \pi_{2 n}^{s}\left(K\left(\mathbb{Z}_{2^{i}}, n-1\right)\right) \rightarrow \pi_{2 n}^{s}\left(K\left(\mathbb{Z}_{2}, n\right)\right) \cong \mathbb{Z}_{2}
$$

is an epimorphism.

Proof. It suffices to prove that the following map $S q^{1}: K\left(\mathbb{Z}_{2}, n-1\right) \rightarrow$ $K\left(\mathbb{Z}_{2}, n\right)$ induces an isomorphism on $2 n$-th stable homotopy group. By the calculation in Milgram's book 20], the first group is generated by the class corresponding to $S q^{1}(t) \otimes S q^{1}(t)$ and the second by $s \otimes s$ where $s, t$ are the fundamental classes of the corresponding groups. 
Now what we want follows from the fact that $S q^{1}$ induces a homomorphism mapping $s \bigotimes s$ to $S q^{1}(t) \bigotimes S q^{1}(t)$.

Proposition 3.8. Let $\widetilde{E}_{n+q}$ (q large) be the following 2-stage Postnikov tower. Then there is a map $f: \Sigma^{q} K\left(\mathbb{Z}_{4}, n-1\right) \rightarrow \widetilde{E}_{n+q}$ such that the composite $\left(\Sigma^{q} F_{n-1}(\mathbb{Z}) \rightarrow\right) \Sigma^{q} K(\mathbb{Z}, n-1) \stackrel{\Sigma^{q} \rho_{4}}{\rightarrow} \Sigma^{q} K\left(\mathbb{Z}_{4}, n-1\right) \rightarrow$ $\widetilde{E}_{n+q}$ if $n=1,2(\bmod 4)($ or, $n=0(\bmod 4))$ induces an isomorphism on $\pi_{r}$ where $r=q+2 n$ as above.

(1). $n=2(\bmod 4)$

$$
\begin{aligned}
& K_{r} \quad \stackrel{i_{2}}{\longrightarrow} \quad \widetilde{E}_{n+q} \\
& \downarrow \Pi_{2} \\
& K_{r-2} \times K_{r} \quad \stackrel{i_{1}}{\longrightarrow} \quad E_{n+q} \quad \stackrel{\omega_{2}}{\longrightarrow} \quad K_{r+1} \\
& \downarrow \Pi_{1} \\
& \Sigma^{q} K\left(\mathbb{Z}_{4}, n-1\right) \stackrel{\Sigma^{q} l_{n-1}}{\longrightarrow} K\left(\mathbb{Z}_{4}, q+n-1\right) \stackrel{S q^{n} \times S q^{n+2}}{\longrightarrow} K_{r-1} \times K_{r+1} \\
& \text { where } i_{1}^{*}\left(\omega_{2}\right)=S q^{2} S q^{1} l_{r-2}+S q^{1} l_{r} \text {. } \\
& K_{r} \stackrel{i_{2}}{\longrightarrow} \quad \widetilde{E}_{n+q} \\
& K_{r-2} \stackrel{i_{1}}{\longrightarrow} \quad E_{n+q} \quad \stackrel{\omega_{2}}{\longrightarrow} K_{r+1} \\
& \downarrow \Pi_{1} \\
& \Sigma^{q} K\left(\mathbb{Z}_{4}, n-1\right) \stackrel{\Sigma^{q} l_{n-1}}{\longrightarrow} K\left(\mathbb{Z}_{4}, q+n-1\right) \stackrel{S q^{n}}{\longrightarrow} K_{r-1}
\end{aligned}
$$

where $i_{1}^{*}\left(\omega_{2}\right)=S q^{2} S q^{1} l_{r-2}$.

(3). $n=1(\bmod 4)$

$$
\begin{array}{clll}
K_{r} \stackrel{i_{2}}{\longrightarrow} & \widetilde{E}_{n+q} & & \\
& \downarrow \prod_{2} & & \\
K_{r} \stackrel{i_{1}}{\longrightarrow} & E_{n+q} & \stackrel{\omega_{2}}{\longrightarrow} & K_{r+1} \\
& \downarrow \prod_{1} & & \\
\Sigma^{q} K\left(\mathbb{Z}_{4}, n-1\right) \stackrel{\Sigma^{q} l_{n-1}}{\longrightarrow} & K\left(\mathbb{Z}_{4}, q+n-1\right) & \stackrel{S q^{n+1}}{\longrightarrow} & K_{r}
\end{array}
$$

where $i_{1}^{*}\left(\omega_{2}\right)=S q^{2} l_{r-1}$.

Proof. Denote the tower in the Proposition by $\widetilde{E}_{n+q}\left(\mathbb{Z}_{4}\right)$. Denote by $\widetilde{E}_{n+q}(\mathbb{Z})$ a similar tower in which $K\left(\mathbb{Z}_{4}, n+q-1\right)$ is replaced by $K(\mathbb{Z}, n+$ $q-1)$. By Remark 3.5, it is easy to see that there is a map from $\Sigma^{q} F_{n-1}(\mathbb{Z})$ to $\widetilde{E}_{n+q}(\mathbb{Z})$ which induces an isomorphism on $\pi_{r}$ when $n=$ $0(\bmod 4)$. On the other hand it is not difficult to see that there is a map from the tower $\widetilde{E}_{n+q}(\mathbb{Z})$ to the tower $\widetilde{E}_{n+q}\left(\mathbb{Z}_{4}\right)$ which induces an 
isomorphism on $\pi_{r}$. It remains to prove that the natural map $\sum^{q} \iota_{n-1}$ : $\Sigma^{q} K\left(\mathbb{Z}_{4}, n-1\right) \rightarrow K\left(\mathbb{Z}_{4}, n+q-1\right)$ can be lifted to $\widetilde{E}_{n+q}\left(\mathbb{Z}_{4}\right)$ and the lifting is compatible to that of the map $\Sigma^{q} \iota_{n-1}: \Sigma^{q} K(\mathbb{Z}, n-1) \rightarrow$ $K(\mathbb{Z}, n+q-1)$ to $\widetilde{E}_{n+q}(\mathbb{Z})$.

We will give a proof only for $n=2(\bmod 4)$, the other cases are similar. Consider the fiber inclusion map $h: \Sigma^{q} \Gamma_{q} \rightarrow \Sigma^{q} K\left(Z_{4}, n-1\right)$, we have the following Peterson-Stein formula

$S q^{2} S q^{1} S q_{h}^{n}\left(\Sigma^{q} l_{n-1}\right)+S q^{1} S q_{h}^{n+2}\left(\Sigma^{q} l_{n-1}\right)=h^{*} \Psi\left(\Sigma^{q} l_{n-1}\right) \in H^{r+1}\left(\Sigma^{q} \Gamma_{q}, \mathbb{Z}_{2}\right) / Q$

where $Q=S q^{2} S q^{1}\left(I m h^{*}\right)+S q^{1}\left(I m h^{*}\right)=S q^{1}\left(I m h^{*}\right)$.

By Theorem 4.6 [20] and a familiar diagram chase argument as in the proof of Proposition 2 in Chap.16 [19](see also [23]), we have $\Sigma^{q}(\theta \otimes \theta) \in S q_{h}^{n}\left(\Sigma^{q} l_{n-1}\right)$ and $\Sigma^{q} e^{2} \cup(\theta \otimes \theta) \in S q_{h}^{n+2}\left(\Sigma^{q} l_{n-1}\right)$. It follows easily that $h^{*} \Psi\left(\Sigma^{q} l_{n-1}\right)=0 \in H^{r+1}\left(\sum^{q} \Gamma_{q}, \mathbb{Z}_{2}\right) / Q$. It is not difficult to see from this and a simple computation that $\Psi\left(\Sigma^{q} l_{n-1}\right)=0$ and a lifting can be chosen such that $\omega_{2}$ lies in its kernel.

To complete the proof, note that, as mentioned before, there is a commutative diagram up to homotopy

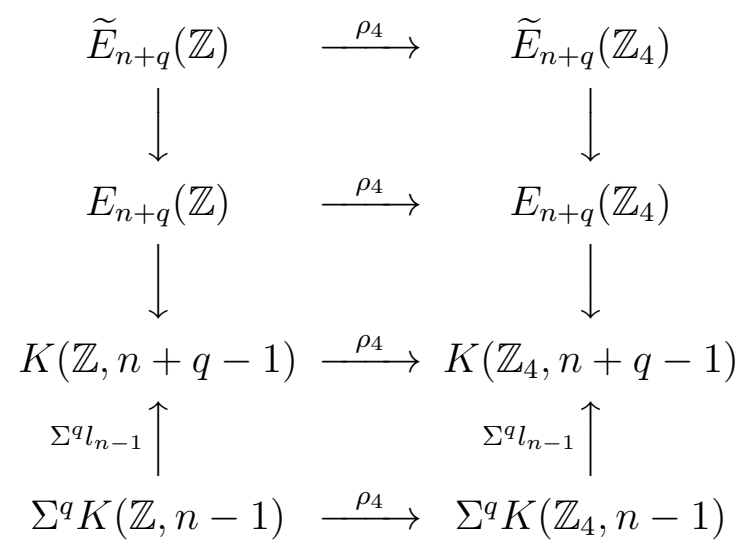

The lifting from $\Sigma^{q} K(\mathbb{Z}, n-1)$ of $\Sigma^{q} l_{n-1}$ and the lifting from $\Sigma^{q} K\left(\mathbb{Z}_{4}, n-\right.$ 1) of $\Sigma^{q} l_{n-1}$ can be made compatible by a modification of the lifting from $\Sigma^{q} K\left(\mathbb{Z}_{4}, n-1\right)$ of $\Sigma^{q} l_{n-1}$. The same way the liftings to $\widetilde{E}_{n+q}$ can also be made compatible. Thus we have the following commutative diagram up to homotopy which completes the proof.

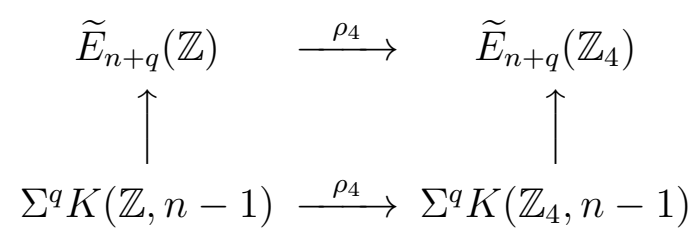


Remark 3.9. The $2^{\text {nd }} \mathrm{k}$-invariant $\omega_{2}$ in the Postnikov tower above gives an unique secondary cohomology operator $\Psi$ (with $Z_{4}$-coefficients) associated with the Adem relation

$$
\begin{array}{ll}
S q^{2} S q^{1} S q^{n}+S q^{1} S q^{n+2}=0 & n=2(\bmod 4) \\
S q^{2} S q^{1} S q^{n}=0 & n=0(\bmod 4) \\
S q^{2} S q^{n+1}=0 & n=1(\bmod 4)
\end{array}
$$

Note that $E_{n+q}$ is the universal example of the operator $\Psi$.

By Peterson-Stein[21], there are operators $\Phi$ which are S-dual to $\Psi$ (which is uniquely determined by $\Psi$ ) so it is a secondary operator associated with the Adem relations:

$$
\begin{array}{ll}
\chi\left(S q^{n}\right) S q^{3}+\chi\left(S q^{n+2}\right) S q^{1}+S q^{1} \chi\left(S q^{n+2}\right)=0 & n=2(\bmod 4) \\
\chi\left(S q^{n}\right) S q^{3}+S q^{1} \chi\left(S q^{n+2}\right)=0 & n=0(\bmod 4) \\
\chi\left(S q^{n+1}\right) S q^{2}+S q^{1} \chi\left(S q^{n+2}\right)=0 & n=1(\bmod 4)
\end{array}
$$

as we stated in $\$ 1$.

\section{Proofs of Theorems 2.3, 2.6 And 2.7}

Proof of Theorem 2.3. First note that it suffices to show this for the universal spectrum $\widetilde{W}(n)$ since the map $i: S^{0} \rightarrow \widetilde{W}(n)$ factors through $i: S^{0} \rightarrow Y$. Notice that $H_{i}\left(\widetilde{W}_{k}(n) / S^{k}\right)=0$ for $i \leq k+2$. Thus in the following proof, we may assume that $Y_{k} / S^{k}$ satisfies the same for $k$ large. Assuming $k$ large, without loss of generality we can assume that $Y_{k}$ is a finite complex. Write $Y_{k}^{*}$ for the $m S$-dual of $Y_{k}$ and $g: Y_{k}^{*} \rightarrow S^{m-k}$ for the $S$-dual of the inclusion $i: S^{k} \rightarrow Y_{k}$. Note that $g^{*}\left(\varsigma_{S^{m-k}}\right) \neq 0$, where $\varsigma_{S^{m-k}}$ is the cohomology fundamental class of the sphere. By the $S$-duality we get a commutative diagram

$$
\begin{array}{cccc}
\left\{S^{2 n+k}, S^{k} \wedge\right. & \left.K\left(\mathbb{Z}_{4}, n-1\right)\right\} & \stackrel{i_{*}}{\longrightarrow} & \left\{S^{2 n+k}, Y_{k} \wedge K\left(\mathbb{Z}_{4}, n-1\right)\right\} \\
\downarrow & & \downarrow & \cong \\
\left\{S^{2 n+m}, S^{m} \wedge K\left(\mathbb{Z}_{4}, n-1\right)\right\} & \stackrel{g^{*}}{\longrightarrow} & \left\{S^{2 n+k} \wedge Y_{k}^{*}, S^{m} \wedge K\left(\mathbb{Z}_{4}, n-1\right)\right\} \\
\downarrow & & \downarrow q_{2 *} \\
{\left[S^{2 n+m}, \widetilde{E}_{n+m}\right]} & \stackrel{g^{*}}{\longrightarrow} & {\left[S^{2 n+k} \wedge Y_{k}^{*}, \widetilde{E}_{n+m}\right]}
\end{array}
$$

where $\widetilde{E}_{m+n}$ is the tower in Proposition 3.8 and $q_{2}: S^{m} \wedge K\left(\mathbb{Z}_{4}, n-1\right) \rightarrow$ $\widetilde{E}_{n+m}$ is a lifting of $\Sigma^{m} l_{n-1}$. From the diagram above and Proposition 3.8, it suffices to show that the homomorphism $g^{*}$ at the bottom line is injective.From now on we will restrict to the case $n \equiv 2(\bmod 4)$. The other cases are similar. Let $i_{0}: F \rightarrow \widetilde{E}_{n+m}$ be the fibre of the composite $\Pi_{1} \circ \Pi_{2}$. Note that $F$ can be viewed as a fibration over $K_{2 n+m-2}$ with fibre $K\left(\mathbb{Z}_{4}, 2 n+m\right)$ and $k$-invariant $j_{*}\left(S q^{2} S q^{1}\right)(l)$; where

$$
j_{*}: H^{m+2 n+1}\left(-, \mathbb{Z}_{2}\right) \rightarrow H^{m+2 n+1}\left(-, \mathbb{Z}_{4}\right)
$$




\section{SECONDARY BROWN-KERVAIRE QUADRATIC FORMS AND $\pi$-MANIFOLDSI7}

is the homomorphism induced by the inclusion $\mathbb{Z}_{2} \subset \mathbb{Z}_{4}$ and $l$ is the basic class of $K_{m+2 n-2}$.

Consider the following commutative diagrams

$$
\begin{aligned}
& {\left[S^{2 n+m}, F\right] \stackrel{\cong}{\longrightarrow} \quad\left[S^{2 n+m}, \widetilde{E}_{n+m}\right]} \\
& \downarrow J:=g^{*} \quad \downarrow g^{*} \\
& {\left[S^{2 n+k} \wedge Y_{k}^{*}, K\left(\mathbb{Z}_{4}, n+m-2\right)\right] \stackrel{i_{1 *}}{\longrightarrow}\left[S^{2 n+k} \wedge Y_{k}^{*}, F\right] \stackrel{i_{0 *}}{\longrightarrow}\left[S^{2 n+k} \wedge Y_{k}^{*}, \widetilde{E}_{n+m}\right]}
\end{aligned}
$$

and

$$
\begin{aligned}
& {\left[S^{2 n+m}, K\left(\mathbb{Z}_{4}, 2 n+m\right)\right] \quad \stackrel{\cong}{\longrightarrow}\left[S^{2 n+m}, F\right]} \\
& \downarrow g^{*} \\
& \downarrow J \\
& {\left[S^{2 n+k} \wedge Y_{k}^{*}, K_{2 n+m-3}\right] \stackrel{j_{*}\left(S q^{2} S q^{1}\right)}{\longrightarrow}\left[S^{2 n+k} \wedge Y_{k}^{*}, K\left(\mathbb{Z}_{4}, 2 n+m\right)\right] \stackrel{\cong}{\longrightarrow}\left[S^{2 n+k} \wedge Y_{k}^{*}, F\right]}
\end{aligned}
$$

where $i_{1}: K\left(\mathbb{Z}_{4}, n+m-2\right) \rightarrow F$ is the homotopy fibre of $i_{0} \cdot j_{*}\left(S q^{2} S q^{1}\right)$ in the second diagram above is zero since $S q^{3} U_{k}=0$ and thus by duality $\chi\left(S q^{3}\right) H^{m-k-3}\left(Y_{k}^{*}\right)=S q^{2} S q^{1} H^{m-k-3}\left(Y_{k}^{*}\right)=0$. Thus the second diagram implies that $J$ is a monomorphism. To complete the proof, it suffices to show $\operatorname{Ker}\left(i_{0}\right)_{*}=\operatorname{Im}\left(i_{1}\right)_{*}=0$ in the first diagram above.

Let $q=m-n-k-1$, if $x \in H^{q-1}\left(Y_{k}^{*}, \mathbb{Z}_{4}\right)$,then $S q^{n}(x) \in H^{n+q-1}\left(Y_{k}^{*}, \mathbb{Z}_{2}\right) \cong$ $\left(H^{k+2}\left(Y_{k}, \mathbb{Z}_{2}\right)\right)^{*}=0$. On the other hand, by duality $\chi\left(S q^{n+2}\right) U_{k}=0$ implies that $S q^{n+2} H^{q-1}\left(Y_{k}^{*}, \mathbb{Z}_{2}\right)=0$. Thus

$$
x \in \operatorname{Ker} S q^{n} \cap \operatorname{Ker} S q^{n+2}
$$

Since $Y_{k}$ is $\Phi$-orientable, i.e, $0 \in \Phi\left(U_{k}\right)$. By [21] that $0 \in \Psi(x)$. Thus $x$ can be lifted to $\widetilde{E}_{q-1}$ and so $\left(i_{1}\right)_{*}(x)=0$. This completes the proof.

For simplicity,denote by $F_{n-1}\left(\mathbb{Z}_{4}\right)$ the space $K\left(\mathbb{Z}_{4}, n-1\right) \wedge K\left(\mathbb{Z}_{4}, n-\right.$ 1) as before in the following proof.

Proof of Theorem 2.6. For $x \in H^{n-1}\left(M, \mathbb{Z}_{4}\right)$, let $f(x)=(w \wedge i d) A_{\alpha}(x) \in$ $H_{2 n}\left(K\left(\mathbb{Z}_{4}, n-1\right) ; \widetilde{W}(n)\right)$. For $k$ large, $f(x+y)$ is the following composition of maps

$$
\begin{aligned}
& S^{1} \wedge S^{2 n+k} \stackrel{i d \wedge \Delta \alpha}{\longrightarrow} S^{1} \wedge T \xi \wedge M_{+} \stackrel{i d \wedge w \wedge(x \times y)}{\longrightarrow} \\
\rightarrow & S^{1} \wedge \widetilde{W}(n)_{k} \wedge\left(K\left(\mathbb{Z}_{4}, n-1\right) \times K\left(\mathbb{Z}_{4}, n-1\right)\right)= \\
= & \widetilde{W}(n)_{k} \wedge S^{1} \wedge\left(K\left(\mathbb{Z}_{4}, n-1\right) \times K\left(\mathbb{Z}_{4}, n-1\right)\right) \stackrel{i d \wedge \kappa}{\longrightarrow} \widetilde{W}(n)_{k} \wedge S^{1} \wedge K\left(\mathbb{Z}_{4}, n-\right.
\end{aligned}
$$
1),

where $\kappa^{*}(l)=l \otimes 1+1 \otimes l$ for the basic class $l \in H^{n-1}\left(K\left(\mathbb{Z}_{4}, n-1\right), \mathbb{Z}_{4}\right)$.

Identifying $\widetilde{W}(n)_{k} \wedge S^{1} \wedge\left(K\left(\mathbb{Z}_{4}, n-1\right) \times K\left(\mathbb{Z}_{4}, n-1\right)\right)$ with

$$
\begin{gathered}
\left\{\widetilde{W}(n)_{k} \wedge S^{1} \wedge K\left(\mathbb{Z}_{4}, n-1\right)\right\} \vee\left\{\widetilde{W}(n)_{k} \wedge S^{1} \wedge K\left(\mathbb{Z}_{4}, n-1\right)\right\} \vee \\
\vee\left\{\widetilde{W}(n)_{k} \wedge S^{1} \wedge F_{n-1}\left(\mathbb{Z}_{4}\right)\right\} .
\end{gathered}
$$

It is readily to see that $f(x+y)=f(x)+f(y)+g$, here $g$ is the composition 


$$
S^{2 n+k+1} \stackrel{i d \wedge \Delta \alpha}{\longrightarrow} S^{1} \wedge T \xi \wedge M_{+} \stackrel{i d \wedge w \wedge \Delta}{\longrightarrow} S^{1} \wedge \widetilde{W}(n)_{k} \wedge M_{+} \wedge M_{+} \stackrel{i d \wedge x \wedge y}{\longrightarrow}
$$

$\widetilde{W}(n)_{k} \wedge S^{1} \wedge K\left(\mathbb{Z}_{4}, n-1\right) \wedge K\left(\mathbb{Z}_{4}, n-1\right) \stackrel{i d \wedge H(\kappa)}{\longrightarrow} \widetilde{W}(n)_{k} \wedge S^{1} \wedge K\left(\mathbb{Z}_{4}, n-1\right)$, where $H(\kappa)$ is the Hopf constuction of $\kappa$.

Now the cofibration

$$
S^{k+1} \wedge F_{n-1}\left(\mathbb{Z}_{4}\right) \stackrel{\Sigma i \wedge i d}{\rightarrow} S^{1} \wedge \widetilde{W}(n)_{k} \wedge F_{n-1}\left(\mathbb{Z}_{4}\right) \rightarrow S^{1} \wedge\left(\widetilde{W}(n)_{k} / S^{k}\right) \wedge F_{n-1}\left(\mathbb{Z}_{4}\right)
$$

is also a fibration at least in the stable range. It follows immediately that

$$
(\Sigma i \wedge i d)_{*}: \pi_{2 n+k+1}\left(S^{k+1} \wedge F_{n-1}\left(\mathbb{Z}_{4}\right)\right) \rightarrow \pi_{2 n+k+1}\left(S^{1} \wedge \widetilde{W}(n)_{k} \wedge F_{n-1}\left(\mathbb{Z}_{4}\right)\right)
$$

is surjective. On the other hand, it is easy to know that the generator $\beta \in \pi_{2 n}^{s}\left(F_{n-1}\left(\mathbb{Z}_{4}\right)\right) \cong \mathbb{Z}_{2}$ satisfies $\beta^{*}\left(l \otimes S q^{2} l\right) \neq 0$. Thus, for the inclusion map $i$, the composition $(\Sigma i \wedge i d) \circ \beta \in \pi_{2 n+k+1}\left(S^{1} \wedge \widetilde{W}(n)_{k} \wedge\right.$ $\left.F_{n-1}\left(\mathbb{Z}_{4}\right)\right)$ induces a nontrivial homomorphism on the $(2 n+k)$-th homology and thus $(\Sigma i \wedge i d)_{*}$ is an isomorphism. Moreover, the generator $g_{0} \in \pi_{2 n}^{s}\left(\widetilde{W}(n)_{k} \wedge F_{n-1}\left(\mathbb{Z}_{4}\right)\right)$ satisfies that $g_{0}^{*}\left(U_{k} \wedge S q^{2} l_{n-1} \wedge l_{n-1}\right) \neq 0$. Thus the composition $(i d \wedge x \wedge y)(w \wedge \Delta)(\Delta \alpha)$ is null homotopy if and only if $\left\langle x \cup S q^{2} y,[M]_{2}\right\rangle=0$. By Proposition 2.1, the proof now follows by the commutative diagram

$$
\begin{array}{cccc}
S^{k} \wedge \Sigma F_{n-1}\left(\mathbb{Z}_{4}\right) & \stackrel{i \wedge i d}{\longrightarrow} & \widetilde{W}(n)_{k} \wedge \Sigma F_{n-1}\left(\mathbb{Z}_{4}\right) \\
& \downarrow i d \wedge H(\kappa) & & \\
S^{k} \wedge \Sigma K\left(\mathbb{Z}_{4}, n-1\right) & \stackrel{i \wedge i d}{\longrightarrow} \widetilde{W}(n)_{k} \wedge \Sigma\left(\kappa K\left(\mathbb{Z}_{4}, n-1\right) .\right.
\end{array}
$$

Proof of Theorem 2.7. Let $\mu: K_{n+k+1} \times \widetilde{W}_{k}(n) \rightarrow \widetilde{W}_{k}(n)$ denote the fiber multiplication. Since $d_{1}\left(w_{1}, w_{2}\right)=0, w_{2}$ is the composition

$$
T \xi \stackrel{\Delta}{\longrightarrow} T \xi \times T \xi \stackrel{w_{1} \times v U_{k}}{\longrightarrow} \widetilde{W}_{k}(n) \times K_{n+k+1} \stackrel{\mu}{\longrightarrow} \widetilde{W}_{k}(n),
$$

where $v U_{k} \in H^{k+n+1}\left(T \xi, Z_{2}\right)$ is the second difference of $w_{1}$ and $w_{2}$, i.e, the secondary obstruction to deform $w_{1}$ to $w_{2}$. Consider the commutative diagram:

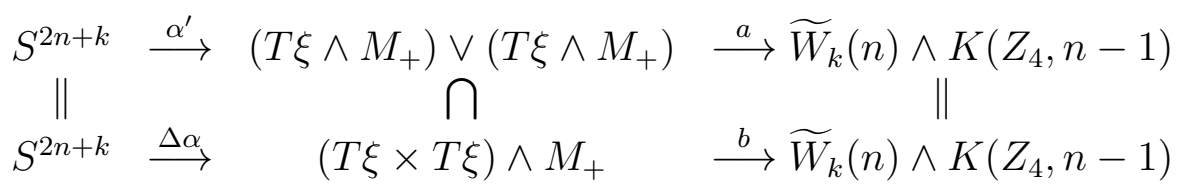

where $\alpha^{\prime}$ is a lifting of $\Delta \alpha, b=\mu\left(w_{1} \times v U_{k}\right) \wedge x, a=\left(w_{1} \wedge x\right) \vee c$, and $c=i\left(v U_{k}\right) \wedge x, i: K_{n+k+1} \rightarrow \widetilde{W}_{k}(n)$ the inclusion of the fibre. Write $\alpha^{\prime}=\alpha_{1}+\alpha_{2}$, here $\alpha_{1}$ and $\alpha_{2}$ are the factors of the wedge. Note that $\phi_{2}(x)=h(b \circ \Delta \alpha)=h\left(a \alpha_{1}\right)+h\left(a \alpha_{2}\right)=\phi_{1}(x)+h\left(a \alpha_{2}\right)$.

We are going to show $h\left(a \alpha_{2}\right)=0$. 
SECONDARY BROWN-KERVAIRE QUADRATIC FORMS AND $\pi$-MANIFOLDS19

As $a \alpha_{2}$ factors through the map $i \wedge i d: K_{n+k+1} \wedge K\left(\mathbb{Z}_{4}, n-1\right) \rightarrow$ $\widetilde{W}_{k}(n) \wedge K\left(\mathbb{Z}_{4}, n-1\right)$, it suffices to prove that

$$
(i \wedge i d)_{*}: \pi_{2 n+k}\left(K_{n+k+1} \wedge K\left(\mathbb{Z}_{4}, n-1\right)\right) \rightarrow \pi_{2 n+k}\left(\widetilde{W}_{k}(n) \wedge K\left(\mathbb{Z}_{4}, n-1\right)\right)
$$

is zero. Note the homomorphism

$$
\left(S q^{1} \wedge i d\right)_{*}: \pi_{2 n+k}\left(K_{n+k} \wedge K\left(\mathbb{Z}_{4}, n-1\right)\right) \rightarrow \pi_{2 n+k}\left(K_{n+k+1} \wedge K\left(\mathbb{Z}_{4}, n-1\right)\right) \cong \mathbb{Z}_{2}
$$

is an isomorphism as it induces an ismomorphism on the $(2 n+k)$-th homology groups. The composition $K_{n+k} \stackrel{S q^{1}}{\longrightarrow} K_{n+k+1} \stackrel{i}{\longrightarrow} \widetilde{W}_{k}(n)$ is null homotopy. Thus $(i \wedge i d)_{*}=0$. This completes the proof.

\section{Proof of Theorem 1.9}

In this section we prove Theorem 1.9. We first study the properties of the invariants $\mu_{M}$ and $q_{M}\left(S q_{i}^{1}\right)$ defined in $\S 1$.

Lemma 5.1. Let $M$ be a framed manifold of dimension $2 n$ with $n$ odd. Let $q_{M}: H^{n}\left(M, \mathbb{Z}_{2}\right) \rightarrow \mathbb{Z}_{2}$ be the Kervaire quadratic form. For $x \in H^{n-1}\left(M, \mathbb{Z}_{2^{i}}\right)$,

(i) $n=3(\bmod 4),[M, x]$ is reduced bordant to zero iff $q_{M}\left(S q_{i}^{1}\right) x=0$.

(ii) $n=1(\bmod 4),[M, x]$ is reduced bordant to $\left[M^{\prime}, x^{\prime}\right]$ where $x^{\prime} \in$ $H^{n-1}\left(M^{\prime}\right)$ iff $q_{M}\left(S q_{i}^{1} x\right)=0$.

Proof. Identify the reduced framed bordism group $\widetilde{\Omega}_{2 n}^{f r}(-)$ with the stable homotopy group $\pi_{2 n}^{s}(-)$. Recall that $\pi_{2 n}^{s}\left(K\left(\mathbb{Z}_{2}, n\right)\right)=\mathbb{Z}_{2}$. By [4] it is easy to see that the homomorphism

$$
\left(S q_{i}^{1}\right)_{*}: \pi_{2 n}^{s}\left(K\left(\mathbb{Z}_{2^{i}}, n-1\right) \rightarrow \pi_{2 n}^{s}\left(K\left(\mathbb{Z}_{2}, n\right)\right)\right.
$$

is identified with the following geometrically defined homomorphism

$$
\begin{array}{ccc}
\widetilde{\Omega}_{2 n}^{f r}\left(K\left(\mathbb{Z}_{2^{i}}, n-1\right)\right) & \rightarrow & \mathbb{Z}_{2} \\
{[M, x]} & \rightarrow & q_{M}\left(S q_{i}^{1}\right) x
\end{array}
$$

By Theorem 3.1 and Lemma 3.7 (i) follows since $\left(S q_{i}^{1}\right)_{*}$ is an isomorphism. To prove (ii), note that there is an exact sequence by Proposition 3.6 and Lemma 3.7

$$
\pi_{2 n}^{s}(K(\mathbb{Z}, n-1)) \rightarrow \pi_{2 n}^{s}\left(K\left(\mathbb{Z}_{2^{i}}, n-1\right)\right) \stackrel{\left(S q_{i}^{1}\right)_{*}}{\longrightarrow} \pi_{2 n}^{s}\left(K_{n}\right)
$$

This completes the proof. 
Now we want to study which bilinear forms $\mu$ can be realized by $(n-$ 2 )-connected $2 n$-dimensional $\pi$-manifolds. Note that a sphere bundle over $S^{n+1}$ with fiber $S^{n-1}$ is a $\pi$-manifold if the characteristic map of the bundle, $\theta \in \pi_{n}(S O(n))$, belongs to the kernel of the stablization homomorphism $S_{*}: \pi_{n}(S O(n)) \rightarrow \pi_{n}(S O)$. Recall that the homotopy groups of $\pi_{n}(S O(n))$ are as follows (c.f: [11]):

$\pi_{n}(S O(n)), n \geq 3, \neq 6$
\begin{tabular}{|c|c|c|c|c|c|c|c|c|}
\hline$n \geq 3, \neq 6$ & $8 s$ & $8 s+1$ & $8 s+2$ & $8 s+3$ & $8 s+4$ & $8 s+5$ & $8 s+6$ & $8 s+7$ \\
\hline$\pi_{n}(S O(n))$ & $\mathbb{Z}_{2} \oplus \mathbb{Z}_{2} \oplus \mathbb{Z}_{2}$ & $\mathbb{Z}_{2} \oplus \mathbb{Z}_{2}$ & $\mathbb{Z}_{4}$ & $\mathbb{Z}$ & $\mathbb{Z}_{2} \oplus \mathbb{Z}_{2}$ & $\mathbb{Z}_{2}$ & $\mathbb{Z}_{4}$ & $\mathbb{Z}$ \\
\hline
\end{tabular}

and $\pi_{6}(S O(6))=0$.

Let $\pi: S O(n) \rightarrow S^{n-1}$ be the canoincal $S O(n-1)$-fiberation. For a $S^{n-1}$-bundle over $S^{n+1}$ with characteristic map $\theta \in \pi_{n}(S O(n))$, say $M_{\theta}$, it is easy to see that $S q^{2}: H^{n-1}\left(M_{\theta}, \mathbb{Z}_{2}\right) \rightarrow H^{n+1}\left(M_{\theta}, \mathbb{Z}_{2}\right)$ is an isomorphism if and only if $\pi_{*}(\theta) \in \pi_{n}\left(S^{n-1}\right)=\mathbb{Z}_{2}$ is nonzero. By duality this implies that $z \cup S q^{2} z=0$ for all $z \in H^{n-1}\left(M_{\theta}, \mathbb{Z}_{2}\right)$ if and only if $\pi_{*}(\theta)=0$. The latter is equivalent to the fact of that the bundle has a section.

Lemma 5.2. Let $M$ be a $\pi$-manifold of dimension $2 n$. Then

(i) $\mu_{M}(x, x)=0, \forall x \in H^{n-1}\left(M, \mathbb{Z}_{2}\right)$ if $n=2(\bmod 4)$.

(ii) $\mu_{M}(x, x)=0, \forall x \in \operatorname{Im}\left(\rho_{2}: T \subset H^{n-1}\left(M, \mathbb{Z}_{4}\right) \rightarrow H^{n-1}\left(M, \mathbb{Z}_{2}\right)\right)$,

if $n$ is odd where $T$ is the set of elements of order 4 .

(iii) If $n=0(\bmod 4)$, then there is a $S^{n-1}$-bundle over $S^{n+1}, M$, so that $\mu_{M}(x, x) \neq 0$, where $x \in H^{n-1}\left(M, \mathbb{Z}_{2}\right)$ is a generator.

Proof. For each $x \in H^{n-1}\left(M, \mathbb{Z}_{2}\right)$, consider the reduced bordism class $[M, x] \in$ $\widetilde{\Omega}_{2 n}^{f r}\left(K_{n-1}\right) \cong \mathbb{Z}_{2}$. It is easy to see that $x \cup S q^{2} x[M]$ is a bordism invariant.

One verifies the following map defines a homomorphism

$$
\begin{array}{ccc}
\widetilde{\Omega}_{2 n}^{f r}\left(K_{n-1}\right) & \rightarrow & \mathbb{Z}_{2} \\
{[M, x]} & \rightarrow & x \cup S q^{2} x[M]
\end{array}
$$

By Remark 3.4 the reduction homomorphism

$$
\widetilde{\Omega}_{2 n}^{f r}(K(\mathbb{Z}, n-1)) \rightarrow \widetilde{\Omega}_{2 n}^{f r}\left(K_{n-1}\right)
$$

is surjective if $n$ is even.

If $n=2(\bmod 4)$, let $\theta \in \pi_{n}(S O(n))$ be a generator. By the tables (I)(II) of [11] it follows that $\theta$ lies in the image of the inclusion map $\pi_{n}(S O(n-1)) \rightarrow$ $\pi_{n}(S O(n))$. By the remark above this implies that the sphere bundle $M_{\theta}$ has a section. Therefore $z \cup S q^{2} z=0$ for all $z \in H^{n-1}\left(M_{\theta}, \mathbb{Z}_{2}\right)$. On the other hand, one can verify that $\left[M_{\theta}, z\right] \in \widetilde{\Omega}_{2 n}^{f r}\left(K_{n-1}\right)$ is a generator if $z \in H^{n-1}\left(M_{\theta}, \mathbb{Z}_{2}\right)$ is nonzero. This proves (i).

If $n=0(\bmod 4)$, by [11] there is an element $\beta \in \operatorname{ker} S_{*}: \pi_{n}(S O(n)) \rightarrow$ $\pi_{n}(S O)$ so that $\pi_{*}(\beta)$ is nonzero. This proves (iii). 
If $n$ is odd, by Lemma 5.1 the homomorphism

$$
\begin{gathered}
q\left(S q^{1}\right): \widetilde{\Omega}_{2 n}^{f r}\left(K_{n-1}\right) \rightarrow \mathbb{Z}_{2} \\
{[M, x] \stackrel{\rightarrow}{\rightarrow} q_{M}\left(S q^{1} x\right)}
\end{gathered}
$$

is an isomorphism. Thus there is a $\delta \in \mathbb{Z}_{2}$ so that $\delta q_{M}\left(S q^{1} x\right)=x \cup S q^{2} x[M]$ for all $[M, x]$. In particular, if $x$ can be lifted to the $\mathbb{Z}_{4}$-coefficient class with order $4, S q^{1} x=0$ and so $x \cup S q^{2} x=0$. This completes the proof.

Now we are ready to prove Theorem 1.9.

Proof of Theorem 1.9. By Theorem 1.6 the data of invariants are homotopy invariants of the manifolds. Thus the homotopy and homeomorphism classification of such manifolds are the same.

There is an isomorphism

$$
\widetilde{\Omega}_{2 n}^{f r}(K(H, n-1)) \cong \pi_{2 n}^{s}(K(H, n-1)) .
$$

Therefore from Theorem 3.1 there is a reduced framed bordism class $[M, f] \in$ $\Omega_{2 n}^{f r}(K(H, n-1))$ corresponding to the given algebraic data $[H, \mu, \phi]$ (resp. $[H, \mu, \phi, \omega]$ ) if $n$ is even (resp. odd). This together with Lemmas 5.1 and 5.2 implies this is an 1-1 correspondence.

Add some $S^{n-1} \times S^{n+1}$ to $M$ if necessary so that $f_{*}: H_{n-1}(M) \rightarrow H$ is surjective. By surgery on $M$ we may assume further that $f_{*}: H_{n-1}(M) \rightarrow H$ is an isomorphism and $H_{n}(M, \mathbb{Q})=0$. Therefore the data can be realized by a $(n-2)$-connected $2 n$-dimensional $\pi$-manifold, $M$, so that $H_{n}(M, \mathbb{Q})=0$ and $\pi(M)=[H, \mu, \phi]$ (resp. $[H, \mu, \phi, \omega]$.

Now it suffices to prove that the map $\pi$ is injective.

Suppose that $M_{i}, i=1,2$, are two framed smooth manifolds with the same data (for TOP manifold, the similar argument works identically). Note that the Kervaire invariants of $M_{i}$ must vanish since $H_{n}\left(M_{i}, \mathbb{Q}\right)=0$. By the assumption there are maps $f_{i}: M_{i} \rightarrow K(H, n-1)$, so that $\left(M_{1}, f_{1}\right)$ and $\left(M_{2}, f_{2}\right)$ are reduced framed bordant, where $f_{i}$ induces an isomorphism on the $(n-1)$-th homology groups. Since both $M_{i}$ framed cobordant to some homtotopy spheres, there is a framed homotopy sphere, $\Sigma$, so that $\left(M_{1}, f_{1}\right)$ and $\left(M_{2} \# \Sigma, f_{2}\right)$ are framed bordant. By Freedman [8] or Kreck [14] it follows that $M_{1}$ and $M_{2} \# \Sigma$ are diffeomorphic since $H_{n}\left(M_{i}, \mathbb{Q}\right)=0$. Therefore $M_{1}$ and $M_{2}$ are almost diffeomorphic. The same argument as above applies to show that $M_{1}$ and $M_{2}$ are homeomorphic to each other. This completes the proof.

Acknowledgements: This paper is a revised version of [7]. This work began during first author's studys and visits at Jilin University, Nankai Institute of Mathematics, Universität Mainz, Universität Bielefeld, the MaxPlanck-Institut für Mathematik and I.H.E.S. He would like to express his sincere thanks to all of those Institutions and to Yifeng Sun and Xueguang Zhou for their encouragements and supports, to Matthias Kreck for teaching him his surgery theory [14]. The second author joins the project at the later 
part of the work mainly for clarifying the argument. Part of the work was done during his visit to Korea University. He would like to thank Prof.Woo Mooha and Department of Mathematics Education for the hospitality.

\section{REFERENCES}

[1] Adams, J.F., On the non-existence of elements of Hopf invariant one, Ann. of Math., 72(1960), 20-104.

[2] Adem, J. and Gilter, S., Secondary characteristic classes and the immersion problem, Bol. Soc. Math. Mexicana., 2(1963),53-78.

[3] Barcus,W.D., The stable suspension of an Eilenberg-Maclane space, Trans. Amer. Math. Soc.,96(1960), 101-113.

[4] Brown, E.H., Generalizations of the Kervaire invariant, Ann. of Math , 95(1972), 368-383.

[5] Browder, W., Surgery on simply connected manifolds, Springer-Verlag, Berlin and New York 1972.

[6] Browder, W., The Kervaire invariant of framing manifolds and its generalizations, Ann. of Math, 90(1969), 157-186.

[7] Fang Fuquan, A new homotopy invariant and its applications , Preprint MPI 96-20.

[8] Freedman, M., Uniqueness theorems for taut submanifolds, , Pac. J. Math., 62(1976), 379-387.

[9] Ishimoto, H., On 3-connected 10-dimensional manifolds, Proc. Japan Academic , 66(1990), 165-168.

[10] Ishimoto, H., On the classification of $(n-2)$-connected $2 n$-dimensional manifolds with torsion free homology groups, Publ. RIMS. Kyoto Univ., 9(1973), 211-260.

[11] Kervaire, M., Some stable homotopy groups of Lie groups, Ill. J. Math , 4(1960), 161-169.

[12] Kervaire, M., A manifold which does not admit any differentiable structure, Comment. Math. Helv, 34(1960), 257-270.

[13] Kervaire, M. and Milnor, J., Groups of homotopy spheres, Ann. of Math , 77(1963), 504-537.

[14] Kreck, M., Surgery and Duality, Ann. of Math., 1999

[15] Levine, J., Lecture on groups of homotopy spheres, Lec. Notes in Math, 1126, $62-95$.

[16] Landweber, P. and Stong, R., A bilinear form for spin manifolds, Trans. Amer. Math. Soc., 300(1987), 625-640.

[17] Mahowald, M. and Williams, R., The stable homotopy of $K(Z, n-1)$, Bol. Soc. Math. Mexicana, 12(1967),22-28.

[18] Mahowald, M. and Peterson, F., Secondary cohomology operations on the Thom class, Topology , 2 (1964), 367-77.

[19] Mosher, R.E. and Tangora, M.C., Cohomology operations and applications in homotopy theory, Harper \& Row, Publishers, 1968.

[20] Milgram, R., Unstable homotopy from the stable point of view, Lec. Notes in Math., 368(1974).

[21] Peterson, F. and Stein, N., The dual of a secondary cohomology operation, Ill. J. Math , 4 (1960), 397-404. 
[22] Peterson, F., and Stein, N., Secondary cohomology operations: Two formulas, Amer. J. Math., 81 (1959), 281-305.

[23] Thomas, E., On functional cup-products and the transgression operator, Arch. Math.,12(1961), 435-444

[24] Wall, C.T.C., Classification problems in differential topology-I, Topology, $\mathbf{2}(1963), 253-261$.

[25] Wall, C.T.C., Classification of $(n-1)$-connected $2 n$-manifolds, Ann. of Math., 75(1962), 163-189.

[26] Whitehead, G.W., Elements in homotopy theory, Springer-Verlag, 1978.

Nankai Institute of Mathematics, Nankai University, Tianjin 300071 , P.R.C

E-mail address: ffang@sun.nankai.edu.cn

Institute of Math.,AcAdemia Sinica ,BeiJing 100080 ,China And

Department of Mathematics Education , Korea University , Seoul , KOREA

E-mail address: pjz62@hotmail.com 